\title{
YTiO3 charge densities: comparison of synchrotron / laboratory diffraction data
}

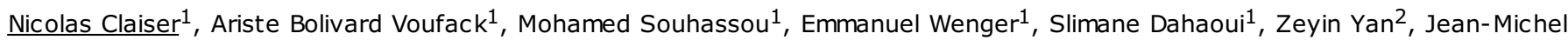
Gillet $^{2}$, Kunihisa Sugimoto ${ }^{3}$, Masa-hisa Ito $^{4}$, Holger Ott $^{5}$

${ }^{1}$ CRM2 / Université De Lorraine, Vandoeuvre-IĖs-nancy, France, ${ }^{2}$ CentraleSupelec, Chatenay-Malabry, France, ${ }^{3}$ JASRI/ Spring-8, Kouto, Japan, ${ }^{4}$ Photon Factory of KEK \& Gunma University, Gunma, Japan, ${ }^{5}$ Bruker AXS GmbH, Karlsruhe, Germany E-mail: nicolas.claiser@univ-lorraine.fr

The title compound, YTiO3, is a perovskite which presents ferromagnetic ordering at low temperature (below 29K). The saturation moment $(0.84 \mu \mathrm{B} . \mathrm{mol}-1)$ corresponds to a single unpaired electron located on the $\mathrm{Ti}$ atom [1]. $\mathrm{A}$ previous neutron diffraction experiment showed that the magnetic structure is in fact more complex, with a main ferromagnetic ordering component along the $c$-axis and two weak antiferromagnetic components along the $a$ and $b$ axes [2]. The present work is part of a more general project aiming to characterize the magnetic pathways using the joint refinement of $X$-ray, polarized neutron diffraction and Compton scattering experiments [3].

Charge density modeling of inorganic materials remains a challenging task due to problems related to the presence of heavy elements; very good or almost perfect crystallinity and high symmetry. Crystal structures with heavy elements have small suitability factor [4] (0.133 in this case) and the good crystallinity induces extinction effects. Moreover, a relatively small unit cell leads to rather few low-order reflections that carry most information concerning valence electrons. Finally, systematic errors in X-ray data can originate from a variety of sources such as beam instability, absorption, integration errors.... To reduce these effects, but also to enhance the intensity of the high-order reflections, the ideal data collection relies on a small size crystal, an intense high-energy monochromatic radiation (very short wavelength) and very low temperature (He) [5]. Two high resolution data were collected, one at $20 \mathrm{~K}$ at Spring8 (BL 02) in the ferromagnetic phase and one at $100 \mathrm{~K}$ in the paramagnetic phase.

This talk will focus on comparing the two data collections but also the resulting charge density model refined.

This work is part of the MTMED (Multi Technique Modelling of Electron Densities) project supported by the French ANR agency. The authors thank Pr. C. Lecomte and Dr. B. Gillon for fruitful discussions and Pr M. Takata for help during the data collection.

[1] J. P. Goral, J. E. Greedan \& D. A. MacLean (1982), J. Solid State Chem. 43, 244-250

[2] C. Ulrich, G. Khaliullin, S. Okamoto, M. Reehuis, A. Ivanov, H. He,Y. Taguchi, Y. Tokura, B. Keimer (2002), Phys. Rev. Lett. 89, 167202-1

[3] Deutsch, M., Gillon, B., Claiser, N., Gillet, J.-M., Lecomte, C. \& Souhassou, M. (2014), IUCr J, 194-199.

[4] E. D. Stevens \& P. Coppens (1976), Acta Cryst. A, 32, $915-917$.

[5] B. B. Iversen, F. K. Larsen, A. A. Pinkerton, A. Martin, A. Darovsky, P. A. Reynolds (1999), Acta Cryst. B, 55, 363 - 374 ;

b) J. Overgaard, B. Schiøtt, F. K. Larsen, A. J. Schultz, J. C. Macdonald, B. B. Iversen (1999), Angew. Chem. Int. Ed., 38, 1239 - 1242.

Keywords: Charge densities - Synchrotron - Perovskite 